\title{
ZUR INSTRUMENTALEN FUNKTION DES RECHTS BEI GESELLSCHAFTLICHEN VERÄNDERUNGEN*
}

\author{
Von Klaus LenK
}

\section{Die Hilflosigkeit der Sozialwissenschaften vor dem Recht}

Sozialwissenschaftler schlagen in der Regel einen Bogen um Erscheinungen, deren Kenntnis und Praxis von der Kaste der Juristen verwaltet werden. Die Analyse von Recht, Rechtsnormen, Rechtsstab, rechtlich geregelten Verfahren fällt aus, weil die meisten Sozialwissenschaftler mit diesen Erscheinungen nicht vertraut sind. Rechtssoziologie, weithin eine Domäne sozialwissenschaftlich dilettierender Juristen, ist nur durch eine Reihe von Juristen-Soziologen zu passablem Stand gelangt.

Können Sozialwissenschaftler nicht umhin, sich dennoch einmal mit rechtlichen Strukturen zu befassen, so erstaunt es gerade den Juristen, mit welcher Unbefangenheit Aussagen von Gesetzestexten, von Gerichten und Rechtswissenschaftlern als Beschreibung der Wirklichkeit mißverstanden werden, obwohl sie als dogmatische Aussagen ihren Stellenwert erst im Kontext bestimmter Entscheidungs- und Argumentationszusammenhänge, Interessenkonstellationen, Gutachten finden. Elemente des Selbstverständnisses von Juristen werden kritiklos übernommen. Diese verstehen Recht, nicht zuletzt zur Aufwertung ihres Berufs und zur Kompensation eines sinkenden Sozialprestiges, als etwas für Bestand und Weiterentwicklung von Gesellschaften Unabdingbares, als Mittel sozialer Kohäsion und sozialer Steuerung zugleich. In Versuche der wissenschaftlichen Anleitung von Gesellschaftssteuerung (policy sciences, sozialistische Leitungswissenschaft, u. a. m.) gehen solche Vorstellungen ungebrochen ein: Recht regle menschliche Beziehungen („social control through law"), Recht könne sozialen Wandel bewirken, es spiele eine Rolle im Übergang zum Sozialismus.

Solches Vertrauen auf die Macht des Rechts ist nicht nur Ursache für die Blindheit gesetzgebender Körperschaften, die eine Erfolgskontrolle der von ihnen beschlossenen Maßnahmen nicht für nötig, möglicherweise gar für schädlich halten. Legalitätsgläubigkeit von links und die Hoffnung, Recht bewähre sich auch als Transmissionsriemen zwischen einer modernisierungswilligen oder revolutionär legitimierten politischen Spitze und dem Beamtenapparat oder der Bevölkerung, sind bezeichnend gerade für die deutsche Szene; auf ihr konnte Legalität (der obrigkeitsstaatliche Rechtsstaat) zum modernen Idealtyp der legitimen Herrschaft avancieren ${ }^{1}$.

Ob Recht tauglich sei, bestimmte Interessen von Minderheiten nicht nur durch Formulierung von Rechtstexten in besonderen Verfahren (Positivierung) ${ }^{2}$ anzuerkennen, sondern sie auch bevorzugt in intendierte gesellschaftliche Zustände zu überführen, ist eine Frage, die außerhalb der Systemperspektive der Agierenden, vornehmlich Juristen, liegt. Eine Überschätzung der Möglichkeiten des Rechts (als

\footnotetext{
* Uberarbeitete Fassung eines Referats für den Arbeitskreis Politische Rechtstheorie der Deutschen Vereinigung für Politische Wissenschaft, Kongreß Duisburg 7. - 10. Oktober 1975.

1 Dazu unten 5.

2 Im Sinne Niklas Luhmanns (Positivität des Rechts als Voraussetzung einer modernen Gesellschaft, in: Die Funktion des Rechts in der modernen Gesellschaft, Jahrbuch für Rechtssoziologie und Rechtstheorie Bd. 1 (1970), S. 175-202).
} 
Kommunikationsmittel, als Auslöser von Motivation, als Träger von Legitimität) kann für ein sozialtechnologisches Interesse an gesellschaftlicher Steuerung fatal werden, gleichviel, ob dieses Interesse sich auf schnellen, revolutionären Wandel, auf Modernisierung vorgeblich rückständiger Gesellschaften oder auf die Ingangsetzung von Reformen richtet, die dazu beitragen können, die weltweite Dominanz ökonomisch begründeter Macht politisch zurückzunehmen und einzugrenzen. Allzu nahe liegt der Verdacht des Wunschdenkens, wenn die, die am Primat „der Wirtschaft" rütteln wollen, in eine Überschätzung des staatlichen Rechts als Steuerungsmittel gegenüber wirtschaftlichen Kräften verfallen. Umgekehrt scheint die Skepsis gegenüber einer solchen Funktion des Rechts von der Befriedigung darüber nicht ungetrübt zu sein, daß den nationalen politischen Systemen signifikante Einbrüche in das wirtschaftliche System verwehrt sind und die Weltgesellschaft bis zu ihrer effektiven Übernahme durch "die Wirtschaft ${ }^{\text {" }}$ steuerungslos driftet. ${ }^{3}$

\section{Klärung des Rechtsbegriffs}

Die Vorstellung, Recht sei eine Sozialtechnologie, lasse sich instrumental einsetzen, beruht auf einer Reihe von Mißverständnissen und Illusionen. Unterschiedliche Funktionen von Recht werden durchweg nicht hinreichend getrennt. Zugrunde liegen Unklarheiten im Rechtsbegriff. Dieser wird sehr verschieden gefaßt, je nachdem, ob Ethnologen gesellschaftliche Normen und Konfliktregelungsprozesse untersuchen wollen, ob Rechtstatsachen oder das „lebende Recht“ erforscht werden sollen, ob Logiker die "Rechtsordnung“ axiomatisieren wollen, ob Politiker sich auf „Recht“ berufen, Rechtsanwälte Ansprüche ihrer Mandanten begründen oder Richter die sonntagsrednerische Überhöhung dessen betreiben, was sie tagtäglich tun. Dementsprechend vielschichtig sind die Aussagen der Rechtsphilosophie und der Rechtstheorie, wobei noch hinzukommt, daß es dort der Erlangung wissenschaftlicher Reputation dienlich ist, altbekannte Zusammenhänge durch neuerfundene Begrifflichkeit zu verfremden.

Die folgende Klärung des Rechtsbegriffs und der Funktionen des (positiven) Rechts strebt keine allgemein verbindliche Definition an. Bei der Vielfalt der Bedeutungen von "Recht" würden hierdurch nur Untersuchungsfelder abgeschnitten. Vielmehr soll verdeutlicht werden, was allen hinter der in der Regel viel zu leichtfertig verwendeten, hochabstrakten Kategorie „Recht“ steht. Welche gesellschaftlichen Beziehungen jeweils als Recht perzipiert und welche ideologischen Vorstellungen damit verbunden werden, ist historisch variabel. Der Kern der Rechtsvorstellungen ist jedoch darauf zurückzuführen, daß gesellschaftliche Verhältnisse dann als Rechtsverhältnisse ins Bewußtsein treten, wenn es zum Konflikt kommt, wenn dieser bewußt ausgetragen wird und wenn besondere Instanzen für die Schlichtung ausgebildet sind.

Um dem Begriff des Rechts Konturen zu verschaffen, sollte man freilich zunächst ein viel breiteres Feld abstecken. Nicht nur aus Gründen der Praktikabilität, auch wegen des erreichten Standes der Differenzierung der Sozialwissenschaften ist es angebracht, einen usurpatorichen Rechtsbegriff zurückzuweisen, auf dessen Grundlage eine Unterscheidung $\mathrm{zwischen}$ Rechtsnormen und sozialen Normen nicht mehr

3 Luhmann a. a. O. S. 301; vgl. auch ders., Rechtssoziologie, 2 Bände, Reinbek 1972, S. 333 ff. 
möglich wäre. Um Recht nicht mit der Gesamtheit der sozialen Strukturen, sozialen Normen und Erwartungen gleichzusetzen, sollte man es auf die bewußtseinsmäßige Repräsentation solcher Strukturen, Normen und Erwartungen beschrän$k^{4}{ }^{4}$. Als Bewußtseinsinhalt wird Recht in der Regel formuliert ${ }^{5}$ und in Texten tradiert.

Dieser - immer noch sehr weite - Rechtsbegriff kann sich auf unterschiedliche Inhalte beziehen, die als Recht ins Bewußtsein gelangen: individuelle und kollektive Erwartungen, vorfindliche Strukturen der Gesellschaft und schließlich Herrschaftsakte, die neue gesellschaftliche Strukturen zu schaffen suchen und von vornherein in Form des (positiven) Rechts auftreten. Hier liegt ein Ansatzpunkt für die Differenzierung des Rechtsbegriffs.

a) Erwartungen, die als Recht bewußt werden, betreffen individuelle und kollektive Bedürfnisse, deren Erfüllung unter gegebenen Produktionsverhältnissen als möglich und daher als gerecht erscheint. Handelt es sich um nicht erfüllte Erwartungen, z. B. von Unterprivilegierten, so haben diese in der Regel nicht die Form des staatlich gesetzten Rechts, es sind keine im Rechtssetzungsprozeß anerkannten Forderungen. Vielmehr erscheinen sie als (subjektives) Naturrecht, Gerechtigkeitsvorstellungen. Hingegen haben staatlich anerkannte (und damit der Erfüllung nähergekommene, wenngleich nicht notwendig erfüllte) Erwartungen die Form des positiven Rechts angenommen.

b) Gesellschaftsstrukturen, z. B. Elemente der Eigentumsordnung, werden aus unterschiedlichem Erkenntnisinteresse als Recht abgebildet. Im Regelfall dient diese Abbildung heute der Legitimierung von (in der Regel richterlichen, nicht notwendig staatlichen) Konfliktentscheidungen aus angeblich geltenden Verhaltensnormen (sozialen Normen); die Kongruenz von Entscheidungsnorm (Maßstab für die Streitentscheidung) und sozialer Norm wird behauptet. Daneben kann sie aber auch $\mathrm{zu}$ ideologisch-argumentativen Zwecken vorgenommen werden, wobei es nicht nur um eine spezifische Berufsideologie von Juristen, sondern auch um das Recht als „Weltanschauung der Bourgeoisie“ (Friedrich Engels) geht.

c) Herrschaftsakte gelten vor allem dann als Recht, wenn sie bestimmte gesellschaftliche Erwartungen anderen Erwartungen vorziehen, indem diese in förmlichen Rechtsetzungsverfahren anerkannt werden und damit zum einen bevorzugte Anwendbarkeit bei der Streitentscheidung erlangen, zum anderen ihren Anspruch auf Befolgung (compliance) auf den Sanktionsapparat des Staats und auf dessen Legitimität stützen können.

Aus jedem dieser Anknüpfungspunkte folgt ein anderer Rechtsbegriff. Ausgehend von individuellen oder kollektiven Erwartungen erscheint Recht vor allem als Rechtsgefühl, subjektives Naturrecht; der Bezug zur Gerechtigkeit steht im Vor-

\footnotetext{
4 Im Gegensatz hierzu bezeichnet die institutionalistische Rechtstheorie die Gesamtheit der gesellschaftlichen Strukturen als Recht, bis hin zu bewußtlos sich durchsetzenden gesellschaftlichen "Naturgesetzen “; eine Unterscheidung zwischen Rechtsnormen und sozialen Normen ist auf dieser Grundlage nicht mehr möglich: ubi societas ibi ius. Eine verwandte Auffassung findet sich bei Eugen Ehrlich, dessen Begriff des lebenden Rechts ${ }^{\alpha}$ nicht nur das von einer Rechtsnorm abweichende wirkliche Verhalten („Rechtswirklichkeit “) umfaßt, sondern die Gesamtheit der sozialen Normen (Eugen Ehrlich, Grundlegung der wirklichkeit ${ }^{\prime}$ ) umfaßt, sondern die Gesamtheit der sozialen Normen (Eugen Ehrlich, Grundlegung der
Soziologie des Rechts (1913), 3. Aufl. Berlin 1967). Für W. G. Sumner (Folkways, Boston 1906) hingegen waren die „Folkways“ nicht selbst Recht, sondern nur dessen Entstehungsursache.

5 Das schließt nicht aus, daß ein bewußt formulierter Rechtssatz unbewußt „befolgt “ wird, d. h., daß ein ihm gleichlaufendes Verhalten tatsächlich existiert.
} 
dergrund. Von den Gesellschaftsstrukturen her zeigt sich das Recht vor allem als objektives (nichtstaatliches, also insofern Natur-) Recht; mit starkem Bezug zur vorherrschenden Ordnung und ihrer Legitimation. Recht als Emanation von Herrschaft schließlich ist vor allem staatliches, positives Recht.

Diese unterschiedlichen Rechtsbegriffe werden oft vermengt. Ist man sich dessen nicht bewußt, so folgen hieraus u. a. Illusionen über eine angebliche „emanzipatorische Funktion" des (positiven) Rechts, sowie eine Überschätzung der Möglichkeit, mit den Mitteln des Rechts sozialen Wandel ingangzusetzen.

\subsection{Subjektives Naturrecht, Rechtsgefühl}

Bedürfnisse, deren Befriedigung als gerecht empfunden wird, werden als Rechtsforderungen ausgedrückt. In Ernst Fraenkels Formulierung ${ }^{6}$ : „Im Rechtsdenken des Proletariats verschwimmen Recht als geltende Herrschaftsordnung und Recht als angestrebter Idealzustand. Das Proletariat glaubt an das Recht. Die Vorstellung, daß eine Rechtsordnung kommen muß, die sich mit der Idee der Gerechtigkeit deckt, ist jeder unterdrückten Gesellschaftsschicht eigen.“

Verschwimmen aber Recht als Herrschaftsordnung und Rechtsgefühl auf diese Weise, so besteht die Gefahr, daß utopische Vorstellungen ins vorhandene Recht hineinprojiziert werden und daß damit oppositionelles Denken für die Legitimation des Bestehenden in Anspruch genommen wird. Die „leere Leidenschaft fürs vorhandene Recht "7 bewirkt, daß unterprivilegierte Klassen das positive Recht, das sie auf weite Strecken von der Befriedigung ihrer Interessen ausschließt, verteidigen als sei es Naturrecht. Dies führt zu empfindlicher Reaktion auf Verletzungen des Status quo, die sich beim Querulanten am deutlichsten zeigt. Wenn Rechtsforderungen nicht verwirklicht werden können, so bedeutet dies Anpassung an eine mächtigere einschränkende Instanz der Außenwelt, „führt zu einem Gleichgewichtszustand, in dem der sich einschränkende seelische Apparat gerade nur das unbedingt nötige Quantum an Verzicht leistet, um dafür die größtmögliche Sicherheit für die übrigen Triebbefriedigungen zu erhalten. Jeder soziale Rechtszustand bedeutet einen solchen Gleichgewichtszustand zwischen Triebverzicht und zugesicherten Befriedigungen, eine Art Vertrag zwischen triebeinschränkenden Instanzen und Triebansprüchen des einzelnen. Der überaus empfindliche gefühlsmäßige Regulator dieses Gleichgewichtszustandes ist das Gefühl der Gerechtigkeit ... Wie die Angst die drohende Gefahr signalisiert, so reagiert der Gerechtigkeitssinn auf jede Bedrohung der erlaubten Triebbefriedigungen, die durch Triebverzichte erkauft wurden ${ }^{8}$.

Hier erklärt sich die Leidenschaft unterprivilegierter Schichten für positives Recht, das sie doch benachteiligt: Wird der Pakt von den Herrschenden gebrochen, das Rechtsgefühl verletzt, so lassen die Triebeinschränkungen nach und ein regressiver Durchbruch verdrängter Bestrebungen erfolgt. Beim Rechtsbruch verliert das Über-Ich seine hemmende Macht über das Ich, und dieses kann nun ungestört den Tendenzen des Es nachgeben. Bei einem relativ geringfügigen Rechtsbruch seitens

\footnotetext{
6 Ernst Fraenkel, Zur Soziologie der Klassenjustiz (1927), Darmstadt 1968, S. 28 ff.

7 Ernst Bloch, Naturrecht und menschliche Würde, Frankfurt 1961, S. 93 ff.

8 Franz Alexander, Hugo Staub, Der Verbrecher und seine Richter, Wien 1929, S. 13.
} 
der Autoritäten werden selbst die tieferen, scheinbar bereits fest organisierten, im Ich aufgenommenen Hemmungen bedroht ${ }^{9}$. Legalität erweist sich in dieser Sicht als das wohlverstandene Interesse der Herrschenden, das Gerechtigkeitsgefühl nicht herauszufordern. Erscheint der Staat als gerecht, so werden Repression und Herrschaft auch ohne Einsicht in ihre Notwendigkeit hingenommen. Eine durchgängige Schlechtbehandlung wird leichter ertragen als einzelne Verletzungen der Gleichheit.

\subsection{Die Darstellung der Gesellschaft als eines Systems von Rechtsbeziehungen; ihre ideologische Funktion}

Recht als Ideologie der bürgerlichen Gesellschaft, als Weltanschauung der Bourgeoisie, erscheint als das gerade Gegenteil des Rechtsgefühls: Legitimation des Bestehenden gegenüber den Rechtsforderungen von Benachteiligten. Ebensowenig aber wie sich Bedürfnisse als Rechtsforderungen ausdrücken müssen, muß sich die bürgerliche Gesellschaft durch Recht legitimieren; freilich stand die Berufung auf Naturrecht am Anfang. Die bürgerliche Gesellschaft ist zunächst juristisch auf ihren Begriff gebracht worden ${ }^{10}$. Soziale Beziehungen stellte man sich vor der Ausdifferenzierung der Soziologie im Wissenschaftssystem als Rechtsbeziehungen vor. Gesellschaft beruht auf Recht. Vor allem mit dem Vertragsbegriff wird die juristische Ideologie zur Ideologie par excellence, wird die theologische Weltanschauung des Mittelalters durch eine zeitgemäßere abgelöst. Das Recht wird Inbegriff der sozialen Beziehungen, die man sich anders als vertragliche nicht mehr vorstellen konnte, bis hin zum Eltern-Kind-Verhältnis und zur Ehe. Das prinzipiell Neue und Fortschrittliche dabei war, daß diese Verhältnisse als von freien Menschen eingegangene und damit kündbar erschienen ${ }^{11}$.

Die Naturrechtslehre der Aufklärung war in weitem Maße eine Darstellung der sozialen Ordnung in verzerrter, unkritischer Weise. Als Vorläuferin der Soziologie glaubte diese Naturrechtslehre, gesellschaftliche Naturgesetze zu entdecken. Die Strukturbeschreibung der Gesellschaft für sozialtechnische, vielfach auch für ideologische Zwecke, ist heute Aufgabe der Soziologie. Das Erkenntnisinteresse der Rechtswissenschaft hat sich mit der wachsenden Arbeitsteilung unter den Sozialwissenschaften präzisiert. Die Rechtswissenschaft beschränkt sich bewußt oder unbewußt auf die Beschreibung jener Teile der Gesellschaftsordnung, die für das Handeln von Gerichten oder anderen Rechtsdurchsetzungsinstanzen als Entscheidungsmaßstäbe von Bedeutung sind. Früher mußten dagegen auch andere Tatbestände in der Sprache des Rechts ausgedrückt werden, da eine adäquatere wissenschaftliche Ausdrucksweise nicht vorhanden war.

Die Bedeutung des Rechts als Ideologie scheint heute zurückzugehen ${ }^{12}$. Die Berufung auf das Recht zu ideologischen Zwecken wird abgelöst durch die Beru-

9 ebd., S. $32 \mathrm{f}$.

10 Jürgen Habermas, Art. „Soziologie“, in: Evang. Staatslexikon, Stuttgart 1966, Sp. 2110.

11 Bernard Willms, Gesellschaftsvertrag und Rollentheorie, in: Die Funktion des Rechts in der modernen Gesellschaft a. a. O., S. 275-298 (281).

12 Freilich besteht die ideologische Funktion des Rechts auch heute noch fort und ist nicht nur für die Sozialisation des Juristennachwuchses von Bedeutung. Die bissige Beschreibung des amerikanischen Rechtsrealisten Thurmann W. Arnold dürfte nach wie vor zutreffen: "Was wir verehrungsvoll Recht nennen, wenn wir allgemein über den Staat sprechen und nicht den Ausgang spezieller Klagen vorhersagen wollen, kann angemessen nur beschrieben werden als eine Art, über den Staat nachzudenken. Es ist eine Art, über menschliche Institutionen in idealistischen termini anstatt in beobachteten Tatsachen zu sprechen. Es befriedigt einen tief verwurzelten Wunsch des Volkes, die staatlichen Einrichtungen möchten als ein schöner Traum erscheinen, in dem, unabhängig vom Menschẹnwerk, 
fung auf das Prestige der Wissenschaften, insbesondere der Sozialwissenschaften; die Umwertung heuristischer Begriffsrahmen zu Bausteinen eines neuen Weltbildes und die Verabsolutierung reduktionistischer Erklärungsmuster sind gleichermaßen bezeichnend hierfür. Die Rechtswissenschaft beschränkt sich in der Regel auf die Strukturbeschreibung von solchen sozialen Gruppen, die Entscheidungsinstanzen ausgebildet haben, für welche gewisse Maßstäbe gesetzt oder gefunden werden müssen. In der Funktion des Rechts, Maßstab der Streitentscheidung zu sein (die Konfliktlösung selbst ist nicht Aufgabe des Rechts, sondern der Rechtsdurchsetzungsinstanzen), liegt das spezifische Erkenntnisinteresse der Rechtswissenschaft bei ihrer Interpretation der sozialen Ordnung ${ }^{13}$. Hier liegt auch das einzig sichere Kriterium für die Unterscheidung des Rechts von Konvention, Brauch und Sitte.

Am deutlichsten ist der ideologische Bedeutungsschwund beim Zivilrecht. Wir sitzen nicht mehr der Vorstellung auf, alle Wirtschaftsbeziehungen seien Rechtsbeziehungen oder beruhten auf solchen. Damit wird auch die in den sich als marxistisch bezeichnenden Versuchen einer Rechtstheorie vorherrschende Tendenz fragwürdig, sich auf die ideologischen Funktionen des Rechts zu konzentrieren. Man scheint eine emanzipatorische Funktion des Rechts retten zu wollen, die in der Naturrechtsideologie der Aufklärung angelegt war, mit dem Abklingen der ideologischen Funktion des Rechts aber verschwinden muß. Die Rede von einer emanzipatorischen Funktion des Rechts ist deswegen verfänglich, weil sie in das positive Recht etwas hineinlegt, was darin nicht enthalten ist. Damit fördert sie Illusionen bei progressiven Außenseitern im Rechtsstab. Wenn der Verlust der ideologischen Funktion des Rechts und damit die Präzisierung des Rechtsbegriffs zudem noch der Rechtstheorie als Schandtat angelastet wird, wenn Kelsen vorgeworfen wird, die Befreiung des Rechts von Ideologie mache es „zum beliebigen Vehikel und schutzlosen Objekt der Machtinteressen“ und „beraubt es seiner Funktion als Kritik an ungerechter Macht und Herrschaft" ${ }^{\text {"14, }}$, so zeigt dies nur die Dringlichkeit einer Klärung des Rechtsbegriffs.

Die ideologische Komponente des Rechtsbegriffs ist auch angesprochen in der Berufung auf Recht in politischen Auseinandersetzungen. Dieser Gebrauch des Rechtsbegriffs scheint ebenfalls nachzulassen, wie sich etwa an der Obsoleszenz weiter Bereiche des Völkerrechts ablesen läßt, die ausschließlich eine ideologische Funktion hatten. Die Innenpolitik der Bundesrepublik Deutschland legt allerdings die Annahme einer „Verrechtlichung der Politik“ nahe, die über die Legitimierung politischer Standpunkte durch Berufung auf den "Rechtsboden“ hinaus eine Auflösung ökonomischer und politischer Beziehungen in Rechtsbeziehungen implizieren soll15. Die Hypothese erscheint dennoch fragwürdig. Es wird auch hier zunehmend deutlich, daß Politik unmittelbar von ökonomischer Macht an die Leine gelegt wird, und nicht so sehr von den Gerechtigkeitsvorstellungen, die von dieser Macht erzeugt werden.

Prinzipien walten ... Teil der Funktion des Rechts ist es, Ideale anzuerkennen, die das genaue Gegenteil des tatsächlichen Verhaltens sind ... Es entwickelt die Struktur einer vollendeten Traumwelt, wo die Logik die Gerechtigkeit schafft. Die Rechtsprinzipien kontrollieren angeblich die Gesellschaft; diese Annahme ist notwendig für die Logik des Traums. Dennoch sollte der Beobachter nicht vergessen, daß die Funktion des Rechts nicht so sehr ist, die Gesellschaft zu führen, als sie zu trösten ${ }^{\alpha}$. (Thurman W. Arnold, Law as Symbolism, in: Vilhelm Aubert (Hrsg.), Sociology of Law, Harmondsworth 1969 , S. 46-51 eigene Übers.)

13 Wenngleich durch das Geschäft der Positivierung (unten 2.3) noch rechtspolitische Funktionen der Rechtswissenschaft hinzukommen.

14 Wolf Paul, Dietrich Böhler, Rechtstheorie als kritische Gesellschaftstheorie, in: Rechtstheorie 1972, S. 75-82 (77). 


\subsection{Das positive Recht und seine Funktion}

Positives Recht ist noch nicht hinreichend dadurch gekennzeichnet, daß es Ausfluß von Herrschaft, sowie formuliertes, in Texte gefaßtes Recht ist. Vor allem ist es das Ergebnis von Verfahren, in denen allgemein akzeptierte Normen oder Erwartungen einzelner gesellschaftlicher Gruppen für verbindlich erklärt werden. Verbindlich heißt dabei, daß die Normen (Erwartungen), die den Filter der Rechtsetzungsverfahren passierten, bevorzugt durch streitentscheidende Instanzen angewandt werden. Ferner, und dies ist der wichtigere Aspekt, können sie zu ihrer Durchsetzung den staatlichen Sanktionsapparat und die Legitimität staatlicher Herrschaft in Anspruch nehmen. Positives Recht ist daher wesentlich auf den Staat bezogen. Es setzt nicht nur voraus, daß Konfliktlösung und bestimmte Sanktionen durch ausdifferenzierte gesellschaftliche Instanzen verwaltet werden, sondern darüber hinaus die Zentralisierung dieser Instanzen beim Staat und die Vorschaltung von besonderen Rechtsetzungsverfahren, auf deren output sich positives Recht im herrschenden Verständnis beschränkt ${ }^{16}$. Positives Recht ist damit weniger das Erzeugnis originärer „Rechtsschöpfung“ als vielmehr das Resultat eines Auswahlprozesses.

Diese Auswahl kann sich darauf beschränken, die Repräsentation der sozialen Ordnung ${ }^{17}$ durch Rechtswissenschaft und Rechtsprechung zu kodifizieren ${ }^{18}$. Dann handelt es sich bei dem daraus entstehenden positiven Recht im wesentlichen um Entscheidungsnormen für richterliche Tätigkeit, die aus sozialen Normen abgezogen sind, welche als allgemein verbindlich betrachtet werden. Durch Kodifizierung soll die Kenntnis der Maßstäbe, nach denen Streit entschieden wird, und damit die Möglichkeit der Berechnung des Ausgangs künftiger Konflikte (Rechtssicherheit) erleichtert werden. Solche Rechtsregeln, die Ausdruck der gesellschaftlichen Ordnung sind, lassen sich als "altes" Recht bezeichnen ${ }^{19}$, im Gegensatz zu jenem ständig anwachsenden Teil des positiven Rechts, der die Steuerungs- und Verwaltungsaufgaben des modernen Staats betrifft ${ }^{20}$, sich vor allem an Verwaltungen, Wirtschaftsorganisationen und einzelne richtet und im Gegensatz zum "alten“ Recht häufig geändert wird.

Das "neue", instrumentale Recht, das "law of social administration" ist das Ergebnis der Positivierung, der Anerkennung von Erwartungen einzelner Gruppen, die - zum Nachteil anderer Erwartungen - den Weg durch die Willensbildungskanäle bis zur politischen Spitze, zur Ministerialbürokratie und zum Parlament "geschafft" haben und nun gestärkt als vorgeblich neutrales Verwaltungsrecht

15 Jürgen Seifert, Verrechtlichte Politik und die Dialektik der marxistischen Rechtstheorie, in: Kritische Justiz 1971, S. 185-200 (186).

16 Die Konkretisierung des Rechts in seiner administrativen und richterlichen Anwendung erscheint dann nicht mehr selbst als Recht. Hier kann auf sich beruhen, ob es für die Zwecke rechtsdogmatischer Systembildung. sinnvoller erscheint, die Beschränkung auf besondere Rechtssetzungsverfahren fallenzulassen und jede Entscheidung staatlicher Funktionsträger als Recht zu bezeichnen; dann müßte freilich auch die enge Verklammerung des Rechtsbegriffs mit dem Normbegriff gelöst werden.

17 also der „folkways“ bzw. des „lebenden Rechts“; vgl. oben Anm. 4.

18 Die Repräsentation kann von der repräsentierten Ordnung abweichen, ohne daß darin die Absicht einer Einwirkung auf diese Ordnung läge, sei es im innovativen oder konservativen Sinne. Positives Recht kann hinter den gesellschaftlichen Verhältnissen herhinken; es kann ihnen aber auch voraus sein, ohne sie doch verändern zu wollen. Vgl. Gerd Winter, Sozialer Wandel durch Rechtsnormen, Berlin 1969, S. 11, Anm. 8.

19 In Anlehnung an W. G. Sumners „law-ways“ spricht Geoffrey Sawer, Law in Society, Oxford 1965, S. 127, von "lawyers' law“, dem Recht, mit dem Justizjuristen vornehmlich zu tun zu haben. Der von Eugen Ehrlich verwendete deutsche Terminus "Juristenrecht" gibt Anlaß zu Mißverständnissen, denen Ehrlich selbst freilich nicht erlegen ist.

20 "Law of social administration“, Sawer ebd. Nur dieses Recht bezeichnet Ehrlich (a. a. O., S. 110, 295 ff.) als staatliches Recht: es kann nur durch den Staat entstehen und bestehen. 
(„im öffentlichen Interesse“21) mit Hilfe des staatlichen Vollzugs- und Repressionsapparats auf gesellschaftliche Verhältnisse zurückwirken können. Neben einem Kernbereich „alten“ Rechts, der zudem von Verwaltungs- und Steuerungsfragen tangiert wird ${ }^{22}$, umfaßt positives Recht vor allem „instrumentales“ (Verwaltungs-) Recht.

Dieses positive Recht kann in verschiedener Weise wirken. Zum einen als Teil der Programmstruktur des Verwaltungshandelns bzw. richterlicher Entscheidungen ${ }^{23}$. Zum anderen kann es unmittelbar bei den Betroffenen durchgesetzt werden, sei es aufgrund der Anwendung durch Sanktionsinstanzen, sei es durch Teilhabe an der motivierenden Kraft staatlicher Autorität, der Würde des Gesetzes. Die Positivierung von Erwartungen kann aber auch eine ganz andere Wirkung aufweisen: Sie kann dazu diesen, die Gruppen zufriedenzustellen, welche Forderungen erhoben haben, ohne daß diese über die symbolische Anerkennung ihrer Forderungen hinaus im Ergebnis etwas gewonnen hätten.

Positives Recht als Anleitung (Programm) des Verwaltungshandelns gibt bindende Maßstäbe für die Anfertigung von Entscheidungen. Hier kann nicht weiter verfolgt werden, inwieweit solche Programme für Verwaltungshandeln und richterliches Handeln wirklich motivierend sind, bzw. inwieweit lediglich die Darstellung von Entscheidungen auf sie abgestimmt wird ${ }^{24}$. Zugrunde liegt ein Problem der Befolgung solchen Rechts durch den Rechts- und Verwaltungsstab, das dem weiter unten diskutierten Problem der Befolgung bzw. Umgehung durch Adressaten („die Wirtschaft", einzelne „Rechtsgenossen“) durchaus parallel liegt, wenngleich einige Besonderheiten der Motivationslage des Personals im öffentlichen Dienst zu beachten sind.

Neben seiner Programmfunktion für öffentliche. Verwaltung und Justiz erhebt positives Recht den Anspruch, das Verhalten der Betroffenen unmittelbar zu beeinflussen: es will befolgt werden. Positives Recht wird als Instrument gesellschaftlicher Steuerung gefaßt, sei es, um sozialen Wandel in gang zu setzen, sei es auch, um ihn zu verhindern. Hier wird die These vertreten, daß es nicht mehr als eine Metapher ist, wenn dabei von einer instrumentalen Funktion des Rechts, von Verhaltenslenkung durch Recht gesprochen wird. Rechtstexte bewirken zunächst nicht mehr, als daß sie ihren Adressaten bestimmte Verhaltensanforderungen übermitteln. Sie sind Kommunikationsmittel, soweit sie neue Verhaltensanforderungen mitteilen, denen das bislang geübte Verhalten nicht entspricht. Aber auch soweit sie lediglich anerkannte soziale Normen kodifizieren, aus ihnen Entscheidungsnormen abziehen, sind es nicht die Entscheidungsnormen, die befolgt werden, sondern die zugrunde liegenden sozialen Normen, das „lebende Recht“ im Sinne Eugen Ehrlichs.

21 Walter Schmidt, Organisierte Einwirkungen auf die Verwaltung, in: Veröff. der Vereinigung der Deutschen Staatsrechtslehrer H. 33 (1974), S. 194 ff., 198: „Das , Offentliche' eines Interesses ist mithin die durch die rechtsverbindliche Entscheidung staatlicher Organe erlangte Zusatzqualität eines ursprünglichen Partikularinteresses."

22 Immer öfter sind Entscheidungsnormen nicht mehr kodifiziertes Juristenrecht, sondern staatliches Recht im Sinne Ehrlichs: Anweisungen des Staats an die Gerichte, wie sie verfahren sollen (Ehrlich a. a. O., S. 152).

23 Die Funktion des positiven Rechts als Maßstab für gerichtliche Streitentscheidungen ist nur ein Sonderfall dieser programmierenden Funktion positiven Rechts.

24 Die Programmbindung ist jedenfalls ein wichtiges Mittel der Legitimation des Verwaltungshandelns. Es wird um so bedeutsamer, je mehr die Auswirkungen solchen Handelns in Widerspruch zu Machtpositionen oder zu eingefahrenen Verhaltensweisen bei den Betroffenen selbst geraten. 


\section{Verhaltenssteuerung durch Recht?}

"Recht als Steuerungsinstrument", die instrumentale Funktion des Rechtes, besagt mithin nicht mehr als daß die Verhaltensanforderungen des positiven Rechts typischerweise auf bestimmte Dispositionen zur Befolgung treffen und daher die Chance besteht, daß sie ein bestimmtes Verhalten der Adressaten auslösen. Die Unterstellung, daß Rechtstexte Verhalten beeinflussen, daß sie eine Form der Kommunikation darstellen, die - wie der Befehl— dem Empfänger nur die Wahl läßt, sein Verhalten danach auszurichten ${ }^{25}$, mag sich bei näherem Hinsehen als notwendige Illusion des Rechtsstabs und des Verwaltungspersonals einer historischen Epoche erweisen, in der zentrale gesellschaftliche Steuerungsinstanzen das Vakuum ausfüllen müssen, das der Zusammenbruch überschaubarer Systeme sozialer Sicherung und sozialer Kontrolle (Familie, Nachbarschaft, Dorf) seit Beginn der Industrialisierung entstehen ließ.

Sie ist aber auch eine Illusion der Politik. Weit verbreitet ist die Auffassung, daß Recht ein von der jeweiligen politischen Konstellation unabhängiges Mittel zur Ingangsetzung sozialen Wandels sei, oder doch gegenüber den gesellschaftlichen und politischen Kräften ein zusätzliches Moment darstelle.

Dabei wird von der Optik des Juristen ausgegangen. Dieser sieht soziale Kontrolle dadurch verwirklicht, daß Gerichte Recht anwenden. Die Gerichtsperspektive des Juristen ließ die Illusion einer sozialen Kontrolle durch Recht aufkommen, die zu einer Überschätzung der Bedeutung von Rechtssetzungsinstanzen, Rechtdurchsetzungsinstanzen und Rechtstexten für die Aufrechterhaltung der sozialen Ordnung führte. Diese Überschätzung kann dann verhängnisvoll werden, wenn ein politisch bewußter Gesetzgeber neue Verhaltensweisen durchsetzen will, die von den Betroffenen nicht ohne weiteres akzeptiert werden. Die oft anzutreffende Auffassung, daß alles menschliche Verhalten — oder doch ein wesentlicher Teil davon - durch Rechtssätze beherrscht oder kontrolliert werde, beruht auf mangelnder Unterscheidung zwischen formulierten Rechtssätzen, also positivem Recht, und den sozialen Normen, nach denen Handeln sich tatsächlich ausrichtet. Man unterstellt, die in Worte gefaßten Rechtsregeln unserer Gesellschaft beeinflußten oder beherrschten unser Verhalten. Diese Annahme erweist sich jedoch als notwendige Illusion von Justizjuristen, die das Verhalten, das sie in ihren Urteilen fordern, damit rechtfertigen, daß es ohnehin herrsche. Die Verwechslung von Entscheidungsregeln, also Regeln, die von Gerichten angewendet werden, mit Verhaltensregeln (sozialen Normen) denen Wirksamkeit,faktische Geltung zukommt, ist notwendige Ideologie des Justizbetriebes, die so lange fortbesteht, wie die Legitimation der Entscheidungen ihrer nicht entbehren kann.

Wie gesagt, ist sie nicht auf die Justiz beschränkt, sondern infiziert auch die Politik. Der Glaube an die Fähigkeit rechtförmiger Texte, gesellschaftliche Verhältnisse in Bewegung zu setzen, entsteht, wie Karl Renner bemerkte ${ }^{26}$, wenn die menschliche Gesellschaft sich ihrer selbst als Gesellschaft bewußt wird und sich zum

25 Maria Borucka-Arctowa, Die gesellschaftliche Wirkung des Rechts (1967), Berlin 1975, S. 87 ff., kennt noch eine Motivationswirkung der Rechtsnorm unmittelbar für das Verhalten der Adressaten. Sie beschränkt dies freilich auf "einige von ihnen... wie ein Steuerbescheid, eine Anweisung zur Ablieferung fremder Valuta, ein "Gestellungsbefehl, ein Gebot zur Vornahme einer polizeilichen Anmeldung usw.“ (S. 89).

26 Karl Renner, Die Rechtsinstitute des Privatrechts und ihre soziale Funktion (Neubearb. 1928), Stuttgart 1965, S. 179. 
Herrn ihrer Umwelt machen will; nach siegreichen Revolutionen besonders stark. Bei gesellschaftlichen Umwälzungen, die bewußt von einer politischen Klasse initiiert werden, kann dieser Wunderglaube an die Gesetzgebung fatal werden, wenn der Bedarf an neuen Verhaltensformen groß ist und die alten juristischen Illusionen weiter mitgeschleppt werden.

Der Oktoberrevolution ist der Glaube an die Allmacht von Dekreten („Dekretinismus") besonders verhängnisvoll geworden. Lenin sah zwar sehr realistisch in Dekreten einen Aufruf an die Massen zum praktischen Handeln. Mehr sind sie in der Tat nicht. Bei Juristen der Revolutionszeit wie Stutschka geht Lenins Einsicht allerdings verloren. Stutschka sieht wohl, daß manche Dekrete wiederholt erlassen werden mußten, sie erst nach dem zweiten oder dritten Erlaß begannen, Geltung zu entfalten ${ }^{27}$. Dennoch stellt sich bei ihm die Illusion ein, mit der Schaffung von Gesetzen sei ein wichtiger Schritt getan, die Dekrete seien die „organisierte Revolution". In den Dekreten sieht er den Überbau eines grundlegenden substantiellen Umschwungs ${ }^{28}$.

Solcher Dekretglaube, der den Dekreten Bedeutung beimißt unabhängig von ihrer Durchsetzung durch Verwaltung und Justiz, bescheidet sich entweder bei deren bloß symbolischer Geltung oder wiegt sich in Illusionen. Nicht nur in Ländern der Dritten Welt dürfte er häufig anzutreffen sein. Auch die Ohnmacht des Gesetzgebers in den westlichen Industriestaaten, dessen Macht sich an den gesellschaftlichen Verhältnissen bricht, wird zu gern mit Rechtsillusionen hinwegdiskutiert. Die Warnungen Eugen Ehrlichs ${ }^{29}$ und vieler anderer, ja sogar Max Webers $^{30}$, vor einer Überschätzung des staatlichen Rechts und des Rechtszwanges, haben es bislang nicht vermocht, eine "wissenschaftlich begründete Kunst der Gesetzgebung" (Ehrlich) hervorzubringen, die die Grenzen des staatlichen Handlungsspielraums kennt, ihn aber auch ausschöpft.

Erst empirische Untersuchungen über die Effektivität von „neuem“, instrumentalen Recht, vor allem in Polen und Skandinavien, haben die problemlose Annahme eines Wirkungszusammenhanges zwischen Erlaß einer Rechtsnorm und daraus resultierender Verhaltensänderung destruiert. Die Ergebnisse dieser Forschungen sind freilich kaum verblüffend: sie finden sich u. a. schon in der Schilderung der englischen Fabrikgesetzgebung bei Karl Marx ${ }^{31}$. Wenngleich die empirischen Untersuchungen durchweg relativ bedeutungslose Gesetze betreffen, so zeigen sie, wie die Effektivität neuer Gesetze letztlich auf ihrer Sanktionsgeltung beruht, auf der sichtbaren und effektiven Repression nicht normkonformen Verhaltens, welche die Anpassung des Verhaltens für die Zukunft geraten erscheinen läßt.

Eine oft angeführte Untersuchung über den Grad der Geltung eines neuen Gesetzes betraf das norwegische Hausangestelltengesetz von 1948 ${ }^{32}$. Gefragt wurde, $\mathrm{ob}$ es tatsächlich seine manifeste Funktion (Arbeitszeitregelung) erfüllte und inwieweit die Verwirklichung des gesetzgeberischen Willens abhängig war von Faktoren außerhalb der Gesetzgebung. Die Gründe für die schwache Befolgung des

\footnotetext{
27 Petr. I. Stučka, Die revolutionäre Rolle von Recht und Staat (1921), Frankfurt 1969, S. 139.

28 ebd., S. 153.

29 "Und so kennzeichnet gegenwärtig die Gesetzgebung der naivste Dilettantismus, der sich darüber ganz im klaren ist, daß es genügt, ein Übel gesetzlich zu verbieten, um es aus der Welt zu schaffen" (a. a. O., S. 331).

30 freilich an versteckter Stelle: Wirtschaft und Gesellschaft, Köln 1964, S. 251

31 Karl Marx, Das Kapital, Bd. 1 (Marx-Engels-Werke Bd. 23), S. 253-320.

32 Vilhelm Aubert, Einige soziale Funktionen der Gesetzgebung, in: Kölner Zschr. f. Soziol. u. Sozialpsych., Sonderheft 11: Studien und Materialien zur Rechtssoziologie (1967), S. 284-309. Kürzere engl. Fassung auch in Aubert (Hrsg.), Sociology of Law a. a. O., S. 116-126.
} 
Gesetzes wurden darin gesehen, daß keine Behörde (wie etwa Gewerbeaufsicht) dafür zuständig war, Verstöße aufzudecken und zu verfolgen. Die Initiative war vielmehr den Hausangestellen selbst überlassen, die jedoch aus Unkenntnis und wegen ihrer starken persönlichen Abhängigkeit wenig Neigung zum Prozessieren zeigten. Ihr einziges Sanktionsmittel war die Kündigung, solange genügend Nachfrage nach ihnen bestand. Die einzige Strafbestimmung des Gesetzes sah eine Geldstrafe vor bei wiederholter Übertretung der Arbeitszeitregelung trotz Protests der Geschädigten. Eine solche Strafbestimmung gebe lediglich vor, eine wirksame Durchführung des Gesetzes anzustreben, während in Wirklichkeit die Stellung der Hausfrau als Arbeitgeber nicht angetastet werde.

Das führte zu der Frage nach der wahren Funktion eines solchen Gesetzes. Die Konservativen sahen in ihm eine Kodifikation des bestehenden Zustandes, die Arbeiterpartei dagegen eine Neuordnung. Beide unterstützten das Gesetz und rechtfertigten es mit diesen widersprüchlichen Argumenten im Parlament. Die Strafbestimmung hatte die Aufgabe, beide Parteien zufriedenzustellen. Die Abgeordneten, die die neuordnende Funktion des Gesetzes sahen, waren mit der Strafbestimmung zufrieden, die Konservativen dagegen konnten sich über ihre offensichtliche Unpraktikabilität freuen. Die wichtigste Funktion des untersuchten Hausangestelltengesetzes scheint damit die Entschärfung eines innenpolitischen Konflikts durch ein scheinbar progressives Gesetz gewesen zu sein, das beide Parteien befriedigte.

Der über die klassischen Problemfassungen hinausgehende Ertrag dieser und anderer $^{33}$ empirischer Untersuchungen liegt darin, daß einzelne Bedingungen der Rechtswirksamkeit identifiziert werden können ${ }^{34}$. Bei der Umstellung von Linksauf Rechtsverkehr in Schweden wurden sozialwissenschaftliche Erkenntnisse über die Wirksamkeit von Rechtsnormen berücksichtigt.

Der Nutzen der Untersuchungen beschränkt sich freilich auf Verhaltensanforderungen, die den isolierten, machtlosen Bürger treffen. Uber die Wirksamkeit von Gesetzen, die auf komplexere Handlungssituationen treffen, vor allem Wirtschaftsgesetze, ist wenig bekannt, was ihre Effektivität steigen könnte. Auch dort, wo traditionelle Moralvorstellungen und Gewohnheiten das Handeln beherrschen, entgleisen die Reformabsichten von intellektuellen Minderheiten oder Kolonialmächten, wie Versuche der Reform mohammedanischen Familienrechts in Israel, der Türkei und der Sow jetunion zeigen ${ }^{35}$.

\footnotetext{
33 Britt-Mari Persson Blegvad, Jette Möller Nielsen, Recht als Mittel des sozialen Wandels - Eine Fallstudie, in: Zur Effektivität des Rechts, Jahrbuch für Rechtssoziologie und Rechtstheorie Bd. 3 (1972), S. 428-446; Donald Campbell, H. Laurence Ross, The Connecticut Crackdown on Speeding: Time-Series Data in Quasi-Experimental Analysis, in: Law and Society Review 3 (1968), S. 33 ff., dies. und Gene V. Glass, Determining the Social Effects of a Legal Reform, in: American Behavioral Scientist 13 (1970), S. 493-509; Richard D. Schwartz, Sonya Orleans, On Legal Sanctions, in: Lawrence M. Friedmann, Steward Macauley, Law and the Behavioral Sciences, Indianapolis 1969, 249262; Johannes Feest, Compliance with Legal Regulations, Observation of Stop-Sign Behavior, in: Law and Society Review 2 (1968), S. 447-461. Uber weitere Untersuchungen berichtet Klaus A. Ziegert, Zur Effektivität der Rechtssoziologie: die Rekonstruktion der Gesellschaft durch Recht, Stuttgart 1975 , S. $94 \mathrm{ff}$

34 Z. B. Karl-Dieter Opp, Einige Bedingungen für die Befolgung von Gesetzen, Kriminologisches Journal 3 (1971), S. 1-25; ders., Soziologie im Recht, Reinbek 1973, S. 90-107; Borucka-Arctowa a. a. O.; Klaus Grimmer, Die Rechtsfiguren einer „Normativität des Faktischen“, Berlin 1971.

35 In Israel wurde die Heraufsetzung des Heiratsalters von der Bevölkerung damit beantwortet, daß Heiraten nicht mehr beim Standesbeamten registriert wurden. Yehezkel Dror, Law and Social Change, in: Aubert a. a. O., S. $95 \mathrm{ff}$.
} 


\section{Bedingungen der Rechtswirksamkeit}

Die Frage, ob Gesetze ihr Ziel erreichen, ist - so gestellt - kaum zu beantworten. Selbst wenn ein Gesetz befolgt wird, ist nicht sicher, ob es das erreichen kann, was der Gesetzgeber bezweckt hat. Die Theorie der Rechtswirksamkeit, der Effektivität des Rechts, wie sie in verschiedenen Skizzierungen in der Rechtssoziologie immer wieder auftaucht ${ }^{36}$, ist in Wahrheit eine Theorie der Befolgung des Rechts, eine Theorie des rechtsrelevanten Verhaltens. Vieles, was bei der Rechtssetzung in westlichen Industriestaaten heute mit $\mathrm{zu}$ beachten wäre, kann sie nicht erfassen.

So sieht sie davon ab, daß gerade "neues“ Recht, Verwaltungsgesetze, oft nicht auf Befolgung durch Individuen angelegt ist, sondern die Allokation von Ressourcen durch Anweisungen an den Staatsapparat bezweckt ${ }^{37}$. Aber auch bei Gesetzen, die ein Verhalten vorzuschreiben scheinen, ist ein aktives Verhalten der Betroffenen oft nicht mehr erforderlich. So bei "automatischer" Rechtanwendung, wenn z. B. Arbeitnehmer ihre Lohnsteuerpflicht dadurch erfüllen, daß ihre Lohnsteuer beim Arbeitgeber ohne ihr Mitwirken abgezogen wird. Gleichgelagert ist der Fall der Realisierung der Norminhalts durch Ersatzvornahme im Verwaltungsrecht.

Schwerwiegender ist das durchgängige Absehen davon, daß die Intentionen des Gesetzgebers nicht einheitlich sind, wie gerade die Untersuchung des norwegischen Hausangestelltengesetzes gezeigt hat. Die Feststellung eines einheitlichen Gesetzzweckes ist vielfach nicht möglich. Gemeinsam ist den Mitgliedern einer gesetzgebenden Körperschaft oftmals nur das Bestreben, eine Frage vom Tisch zu bringen, sich von politischem Druck zu entlasten. Oft ist auch nur die symbolische Geltung eines Gesetzes bezweckt; es hat dann nur die Funktion, soziale Ideale $\mathrm{zu}$ verkünden und ist gar nicht darauf angelegt, wirksam $\mathrm{zu}$ werden ${ }^{33}$. Positives Recht enhält hier ideologische Funktion, was sich etwa in dem Glauben ausdrückt, mit Grundrechten mehr bewirken zu können als Schlupfwinkel des gestrigen gemäß den proklamierten Grundsätzen des bereits im Prinzip Erreichten auszuräuchern.

Die Ausblendung des Positivierungsvorganges, des Rechtssetzungsprozesses, führt dazu, daß der Theorie der Rechtswirksamkeit nur dann ein Erklärungswert zukommt, wenn der Einfluß derer, die ein Gesetz zu befolgen haben werden, auf den Positivierungsprozeß vernachlässigt werden kann. Dies ist der Fall, wenn die Betroffenen isolierte Individuen ohne spezifisch einsetzbare Machtressourcen sind. Mächtige Interessenten werden in sozialen Normallagen ihren Einfluß bereits bei der Rechtssetzung geltend machen, und nicht durch Umgehungsstrategien und Gehorsamsverweigerung gegenüber einmal bestehenden Normen. Im Hinblick auf eine der wichtigsten Fragen - politische Kontrolle über die Wirtschaft - sinkt damit der Erklärungswert einer Theorie der Rechtswiksamkeit. In sozialen Nomallagen ist das Problem der politischen Kontrolle der Wirtschaft keine Frage der Rechts-

\footnotetext{
36 Hans Ryffel, Rechtssoziologie, Neuwied 1974, S. 243 ff.; ders., Bedingende Faktoren der Effektivität des Rechts, in: Zur Effektivität des Rechts a. a. O., S. 225 ff.; Claus Ott, Die soziale Effektivität des Rechts bei der politischen Kontrolle der Wirtschaft, ebd., S. $345 \mathrm{ff}$.; Lawrence M. Friedman, Einige Bemerkungen über eine allgemeine Theorie des rechtsrelevanten Verhaltens, ebd., S. $206 \mathrm{ff}$.

37 Vilhelm Aubert, The Social Function of Law, in: Die Funktionen des Rechts, ARSP Beiheft NF 8, Wiesbaden 1974, S. 85-97 (92 f.).

38 Peter Noll, Gründe für die soziale Unwirksamkeit von Gesetzen, in: Zur Effektivität des Rechts, a. a. O., S. $259 \mathrm{ff}$. (261); Friedman a. a. O., S. $208 \mathrm{ff}$.
} 
wirksamkeit mehr, sondern eine Frage der Einflußverteilung im Gesetzgebungsprozeß39.

Anders ist es bei bewußten Versuchen zur Initiierung sozialer Reformen durch intellektuelle Minderheiten, Kolonialmächte, oder allgemeiner durch Machtzentren, die dem Konzert der Interessentenstimmen gegenüber taub sind - sei es, weil die institutionalisierten Rückmeldekanäle fehlen, sei es aus bewußter Entscheidung. Soweit ihre Reformen sich auch als Rechtsreformen darstellen, ist eine Theorie der Rechtswirksamkeit von entscheidender Bedeutung. Solange es sich nicht nur um die Anpassung rückständiger Institutionen an eine im Grunde bereits vollzogene Umwälzung geht, bleibt der Ausgang des Versuches, beschleunigten (revolutionären) sozialen Wandel in Gang zu setzen, ungewiß, gleichviel, ob die Gegenkräfte traditionalistischer Art sind oder von wirtschaftlich begründeten Machtpositionen und Profitinteressen bzw. privilegierten Bürokratien verkörpert werden.

Wenngleich die rechtssoziologischen Untersuchungen die naive Annahme vom Recht als einem nahezu unbegrenzt einsatzfähigen Instrument zur Ingangsetzung sozialen Wandels zurückgewiesen haben, ist die Frage nach den Bedingungen der Effektivität des Rechts keineswegs zureichend geklärt; Operationalisierungen haben mit beträchtlichen Schwierigkeiten zu kämpfen ${ }^{40}$. Die empirischen Untersuchungen legen nahe, daß mindestens die Probleme der Information des Adressaten und seiner Bereitschaft zu einem vorschriftskonformen Verhalten erörtert werden müssen. Der Bürger muß über ein Gesetz informiert sein, das er einhalten soll, wenn er nicht ohnehin schon tut, was das Gesetz erfordert. Er muß ferner zu gesetzeskonformem Verhalten bereit und imstande sein. Die bloße Übermittlung von Rechtstexten erreicht an sich noch keine Bereitschaft des Empfängers, sich entsprechend $\mathrm{zu}$ verhalten. $\mathrm{Da}$ in Rechtstexten dennoch gemeinhin mehr als ein bloßes Kommunikationsmittel gesehen wird, liegt daran, daß in vielen Fällen der Adressat bereit ist, entweder gerade diese Kommunikation oder aber im großen und ganzen alle Kommunikationen des "Senders“ hinzunehmen, ordnungsgemäß zuzustande gekommenen Rechtsregeln Gehorsam zu erweisen. Die Bereitschaft hierzu kann erfaßt werden als Resultante aus dem Sanktionsdruck, der vom Staat oder von anderen Stellen ausgeht, sowie der Anerkennung (Legitimität) des Staates bzw. einzelner Rechtsregeln ${ }^{41}$.

Die Probleme der Kommunikation zwischen Gesetzgeber und Adressaten können hier nur angedeutet werden; sie werden inzwischen recht häufig diskutiert. Gesetze, deren Adressaten nicht die Staatsbürokratie oder Wirtschaftsorganisationen sind,

39 Ott a. a. O., S. 407.

40 Karl-Dieter Opp, sowie die Diskussion zwischen Opp u. a. im Kriminologischen Journal 7 (1975), S. $99 \mathrm{ff} ., 182 \mathrm{ff} ., 210 \mathrm{ff} ., 291 \mathrm{ff}$.

41 Nichts anderes ergibt sich aus den Theorien der (faktischen) Geltung des positiven Rechts. Macht und Anerkennung sind die jeweils extremen Realisierungsmöglichkeiten des Geltungsgrunds des Rechts. Im Regelfall ergänzen und bedingen sie sich wechselseitig (Martin Drath, Grund und Grenzen der Verbindlichkeit des Rechts, 1963, S. 40). Die Gleichsetzung von Geltung und Wirksamkeit des positiven Rechts wird jedoch in der Rechtstheorie durchweg nicht auf einzelne Rechtsnormen, sondern auf die Rechtsordnung im ganzen bezogen: Sobald die Rechtsordnung insgesamt wirksam ist (= der Staat nicht zusammengebrochen ist), kommt es nur noch darauf an, ob eine Rechtsnorm zu dieser Ordnung gehört, was durch bestimmte Regeln (rules of recognition, H. L. A. Hart, The Concept of Law, Oxford 1961, S. 89 ff.) festgelegt wird. Auf diese Frage wird beträchtlicher Scharfsinn. verwandt. Dadurch wird die Frage nach der Verbindlichkeit des Rechts an der Grenze der Zuständigkeit des Juristen abgeschnitten. Die Illusion einer eigenständigen „juristischen“ Geltung des Rechts neben seiner faktischen Geltung entsteht, unter Umständen noch überhöht durch eine "normative“ Geltung; in letztere gehen herrschende Gerechtigkeitsvorstellungen ein, vor allem solche, die innertung; in letztere gehen herrschende Gerechtigkeitsvorstellungen ein, vor allem solche, die innerzur Illusion einer quasi automatischen Anwendung aller Gesetze durch Justiz und öffentliche Verwaltung. Die Befolgung (durch entsprechendes Verhalten) seitens des Rechtsstabs und der Verwaltungsbürokratie wird damit unterstellt; Nichtanwendung von Gesetzen durch staatliche Instanzen erscheint als pathologische Ausnahme. Das Effektivitätsproblem jenes Teils des Rechts, das sich an staatliche Instanzen richtet, wird hinwegdefiniert. 
müssen verständlich sein, bieten dann aber u. U. leichtere Umgehungsmöglichkeiten. Die Verkündung im Gesetzblatt, das wenige lesen, ist nur ein Teil des Kommunikationsprozesses. Massenmedien, Öffentlichkeitsarbeit, mündliche Information spielen ebenso eine Rolle wie Rechtsanwälte und Interessenverbände, die Rechtskenntnisse an ihre Mandanten bzw. Mitglieder weitergeben. Bei der Erörterung der Möglichkeiten, die Information des Adressaten einer Rechtsnorm zu verbessern, bleibt aber weithin ungeklärt, welche Bedeutung seiner Informiertheit für die Befolgung des Gesetzes zukommt. Vermutlich hängt es vom Inhalt einer Vorschrift und damit von ihrer Anerkennung ab, ob bessere Informiertheit $\mathrm{zu}$ einem höheren Grad der Befolgung führt oder eher das Gegenteil bewirkt ${ }^{42}$.

Bei neuem Recht, dem noch keine sozialen Normen entsprechen, muß konformes Verhalten durch Bestehen oder Schaffung einer Situation erreicht werden, aus der die Bereitschaft zur Befolgung resultiert. Aus der Juristenperspektive wird die Rolle der erwarteten Sanktionen hierbei häufig überbetont. Die Angst vor formalen, staatlichen zentralisierten Sanktionen allein reicht nicht aus, um einer Norm Effektivität zu verleihen. Weniger die abschreckende Wirkung der einzelnen Sanktion ist von Bedeutung als vielmehr ihr Beitrag zur Schaffung eines normativen Klimas ${ }^{43}$, in welchem Normen auch ohne innere Anerkennung sozusagen zähneknirschend ins eigene Verhalten übernommen werden und informale soziale Sanktionen dadurch mobilisiert werden, daß der Eindruck entsteht, der staatliche Rechtserzwingungsapparat nehme seine Aufgabe ernst ${ }^{44}$. Wichtig hierfür ist nicht in erster Linie die Schwere der (formalen) Sanktion, sondern die Häufigkeit ihres Eintritts und ihre Nähe. Hieraus lassen sich eine Reihe von Folgerungen ableiten, die allesamt darauf zielen, die Sanktionsdichte zu erhöhen. Sanktionsressourcen sollen nicht für Kleinigkeiten (Bagatellsachen) eingesetzt werden. Der Selektionsstil vor allem der Polizei soll verbessert werden; der Polizist soll nicht mehr von gewissen äußerlichen Erscheinungen einer Person her auf deren abweichendes Verhalten schließen. Information über Normbrüche soll möglichst unverzerrt bis an die Stellen gelangen, die einschreiten müssen. Situationen des Normbruchs sollen möglichst transparent werden; letzteres kann freilich Folgen für den Schutz der „Privatsphäre“ haben: um Kindesmißhandlungen weithin einzuschränken, wäre Schnüffelei in dieser Privatsphäre durchaus angebracht, auch bevor sich ein „Verdacht" ergibt oder jemand beim staatlichen Rechtserzwingungsapparat „in Erscheinung tritt".

Bei neuem instrumentalem Recht ist die Notwendigkeit effizienter Überwachungsund Sanktionsmechanismen weitaus größer als bei altem Recht, das sozialen Normen parallel läuft. Dennoch ist die faktische Geltung von neuem Recht so lange prekär, wie sie vorwiegend auf der Furcht vor Sanktionen beruht. Strategien der Gesetzesumgehung, buchstabengetreue Erfüllung bei gleichzeitiger Vereitelung des vom Gesetzgeber intendierten Zwecks nehmen zu, wenn es neuem Recht nicht gelingt, von seinen Adressaten als verpflichtend anerkannt zu werden. Hierauf konzentriert sich die Erörterung im folgenden ${ }^{45}$.

42 Andreas Diekmann, Eine empirische Uberprüfung der rechtssoziologischen Theorie von Opp, in: Kriminologisches Journal 7 (1975), S. 182-202 (197).

$43 \mathrm{Daß}$ eine allzu große Sanktionsdichte hierfür auch schädlich sein kann, zeigt Heinrich Popitz, Uber die Präventivwirkung des Nichtwissens, Tübingen 1968.

44 Richard G. Salem, William J. Bowers, Severity of Formal Sanctions as a Deterrent to Deviant Behavior, in: Law and Society Review 5 (1970), S. 21-40.

45 Vernachlässigt wird, daß der Bürger nicht nur zu normkonformen Verhalten bereit, sondern auch in der Lage sein muß, vgl. Friedman a. a. O., S. 215. 


\section{Die Anerkennung neuen Rechts}

$\mathrm{Zu}$ klären ist zunächst, was unter Anerkennung verstanden werden soll. Die Verinnerlichung einer Norm aus reiner Überzeugung würde den Gegenpol zur bloßen Befolgung aus nackter Angst vor Sanktionen markieren. Würde man aber den Anerkennungsbegriff so eng fassen, so wäre es schwierig, die Grauzone teilnahmsloser Hinnahme neuen Rechts zwischen den genannten Extrempunkten zu diskutieren, das normative Klima der Rechtswirksamkeit weiter zu präzisieren. Hier soll daher unter Anerkennung jede Bereitschaft verstanden werden, neues Recht nicht nur aus Angst vor Sanktionen zur Verhaltensgrundlage zu machen.

Nicht geklärt werden kann hier, auf welchen Wegen neues Recht zur Verhaltensgrundlage wird, wie sich seine Anerkennung vollzieht. Wirkungshypothesen wie Podgóreckis Dreistufenmodell46 gehen davon aus, daß die Anerkennung (Legitimität) des Staates bzw. einzelner Rechtsnormen nicht aus der Persönlichkeitsstruktur der Bürger allein erklärt werden kann, sondern durch (eine oder mehrere) Schichten rechtlicher Subkultur (Gruppen, Organisationen) vermittelt ist. Ahnliches besagt die Auffassung, Recht gehe zunächst durch das Medium der öffentlichen Meinung, bevor es von den Adressaten anerkannt werde.

Hiervon zu trennen ist die Frage nach den Ursachen der Anerkennung neuen Rechts. Vermutlich gründet sich die Anerkennung neuen Rechts im wesentlichen auf die folgenden Umstände:

- es wird für vernünftig gehalten,

- die Legitimität des alten Rechts färbt auf es ab,

- es nimmt teil an der Legitimität der Positivierungsinstanz (des Staats).

Eine opinio necessitatis, das Bewußtsein, zur Befolgung verpflichtet zu sein, bildet sich am ehesten, wenn die Adressaten einer Norm diese durchweg für vernünftig halten. Entsprechende Beobachtungen lassen sich im Straßenverkehr machen. Wird ein Verhalten, dessen Gefährlichkeit die meisten noch nicht selbst erlebt haben, verboten, so bildet sich eine solche Überzeugung nicht, und Übertretungen sind entsprechend häufig. Die Notwendigkeit für den Staat, nur noch einsehbar „vernünftige" Normen zu erlassen, nimmt in dem Maße zu, wie die übrigen Legitimitätsquellen neuen Rechts an Wirksamkeit verlieren. Entsprechend wächst die Bedeutung von Erziehung, Aufklärung, Öffentlichkeitsarbeit, Überredung, Mitwirkung der Betroffenen bei der Gesetzesberatung (Partizipation). Nicht von ungefähr ist immer öfter von "Sozialisation durch Recht“ die Rede. Problematisch dabei ist, daß neues Recht oft kurzfristig Effektivität intendiert, die Einsicht in seine Vernünftigkeit sich aber erst nach langer Zeit einstellen mag ${ }^{47}$.

Neben die Vernünftigkeit neuen Rechts im Einzelfall tritt als Anerkennungsgrund eine generalisierte Bereitschaft, alles neue Recht in gewissen Grenzen zu akzeptieren. Zum Teil kann dies darauf zurückzuführen sein, daß neues Recht sich in derselben Form präsentiert wie altes Recht. Dieses schöpft seine Legitimität daraus, $\mathrm{da} ß$ es auf der Übereinstimmung mit sozialen Normen beruht: Es ist traditional legitimiert. Wird altes Recht, das sozialen Normen entspricht, in Texte gefaßt, dann haben diese Texte nur die Funktion der Anleitung der Gerichte (und der Herstellung von Rechtssicherheit), nicht aber (oder nur am Rande) der Motivie-

46 Adam Podgórecki, Dreistufen-Hypothese über die Wirksamkeit des Rechts, in: Studien und Materialien zur Rechtssoziologie a. a. O., S. 271-283.

47 Friedman/Macauley a. a. O., S. 215. 
rung zur Befolgung. Die Legitimität des alten Rechts dehnt sich zu einer allgemeinen Überzeugung aus, daß alle rechtsförmig auftretenden Regelungen anerkannt werden müssen. Neues, instrumentales Recht zehrt damit von dem Legitimitätspotential, das altes, mit wirklichem Verhalten übereinstimmendes Recht akkumuliert hat. Hieran knüpft sich die Hypothese, daß diese Legitimitätsquelle, bei zu starker Inanspruchnahme, sich im Laufe der Zeit erschöpft. Instrumentales Recht, das nicht spontan oder aufgrund entsprechender Erziehung oder Beeinflussung als sinnvoll und damit verpflichtend akzeptiert wird, könnte das vom alten Recht akkumulierte Legitimitätspotential aufzehren ${ }^{48}$.

Weitaus wichtiger jedoch ist die Legitimität der Positivierungsinstanz, des Staates. Sie drückt sich aus in der generalisierten Bereitschaft, alle staatlichen, d. h. zur Rechtsordnung gehörenden, juristisch geltenden Normen zu befolgen, von extremen Ausnahmen abgesehen. Diese Legitimität war so lange problemlos, wie sie sich in der allgemeinen Geneigtheit ausdrückte, Befehlen der Obrigkeit zu gehorchen. Gesetze sind Befehle des Staats; nach ihrem Sinn wird in der Regel nicht gefragt.

In Deutschland dürfte diese Tendenz sich deutlicher als anderswo gezeigt haben, im besonders stark ausgeprägten redlichen Beamtenbewußtsein ${ }^{49}$, aber auch in einer Untertanengesinnung. Nur hier konnte der Gedanke gefaßt werden, den obrigkeitsstaatlichen deutschen. Rechtsstaatsgedanken - die bloße Legitimität durch Legalität - zum modernen Idealtypus der Herrschaft hochzustilisieren ${ }^{50}$. Der im Obrigkeitsstaat gegebenen allgemeinen Geneigtheit, unreflektiert Befehle zu befolgen, entspricht eine imperative Auffassung von Recht, unter der dann tatsächlich ein Wort des Gesetzgebers genügte, um die Fußtruppen des Wandels in Marsch zu setzen, jedenfalls soweit „die Wirtschaft" mitmachte oder voranging. Persson Blegvad und Möller Nielsen sprechen von einer "generellen, wir würden heute sagen: unkritischen Anerkennung des Rechts als Instrument des sozialen Wandels im Nazi-Deutschland ${ }^{51}$ “. Anzumerken wäre dazu allenfalls, daß Legalitätsglaube schichtenspezifisch unterschiedlich stark eingebläut wurde und wird. Vertreter der unteren Schichten im politischen Geschäft sind vermutlich immer noch am ehesten mit der bloß symbolischen Erfüllung ihrer Forderungen durch die Gesetzgebungsmaschinerie zufriedenzustellen.

Wichtiger noch als der Gehorsam der Rechtsgenossen ist die generalisierte Bereitschaft des Personals im öffentlichen Dienst zur lückenlosen Anwendung des Rechts. Das Verhältnis des Beamten zu den Regeln, die er verwaltet, ist weithin durch das jeweilige soziale Klima geprägt, in dem er lebt. Gerade im Einstellungswandel im öffentlichen Dienst läßt sich der Abbau des sozialen Klimas des Obrigkeitsstaats deutlich verfolgen.

Unkritische Hinnahme von Recht, nur weil es Recht ist, Legalität als Legitimitätsgrund neuer Rechtsetzung, ist kein Endzustand. Eher handelt es sich um eine

\footnotetext{
48 Trifft dies zu, so mag der Anreiz entfallen, Wünsche nach verändertem Verhalten, die sich nicht nur an die Staatsbürokratie richten, in die Rechtsform zu kleiden. Pläne, Programme, Appelle an die Ơffentlichkeit würden reichen, soweit nicht gesetzliche Tatbestandsumschreibung für das Handeln von staatlichen Sanktionsinstanzen erforderlich ist.

49 Hierzu Max Webers Diskussionsrede in einer Debatte der Generalversammlung des Vereins für Socialpolitik, Wien 1909, in: Max Weber, Werk und Person, Tübingen 1964, S. $514 \mathrm{ff}$.

50 Max Weber, Wirtschaft und Gesellschaft, 1964, S. $160 \mathrm{ff}$. Ein spezifisch moderner Idealtypus wäre hingegen die Herrschaft, die sich durch effizientes, reibungsloses Funktionieren legitimiert.

51 a. a. O., S. 440 .
} 
„Übergangserscheinung von der Antike zur Moderne52“. Die gelebte Präsenz des Obrigkeitsstaats unterschlug die Einsicht in die Existenz von Mechanismen, die die Befolgung der Rechtsnormen - sowohl durch den öffentlichen Dienst wie durch den einzelnen Rechtsgenossen - im großen und ganzen als unproblematisch erscheinen ließen.

Das Ende unkritischer Hinnahme neuen Rechts signalisiert die Notwendigkeit der Beschaffung von Legitimität, der Legitimation. Die abstrakte Einsicht, es müsse irgendeine staatliche Rechtsordnung geben fördert die Bereitschaft zur Hinnahme beliebiger normativer Inhalte nur in geringem Maße. Wachsendes Bewußtsein von den Verzerrungen repräsentativdemokratischer Willensbildung läßt es geraten erscheinen, die demokratische Legitimation des Gesetzgebers durch Partizipation der Betroffenen bei der Gesetzgebung zu ergänzen. Der Gesetzgeber muß schließlich, wie oben angedeutet, auf seine Autorität bedacht sein, durch wissenschaftliche Beratung oder anderswie die Überzeugung fördern, daß er am besten wisse, was richtig und was falsch sei.

\section{Ergebnis}

Die Umsetzung rechtsförmig kommunizierter Verhaltensanforderungen in gesellschaftlich relevantem Maße in wirkliches Verhalten ist so voraussetzungsvoll, daß das Schlagwort vom sozialen Wandel durch Recht verfehlt erscheint. Soweit Recht nicht nur zufällig über vorherrschende Erwartungen und soziale Normen hinausgeht, läßt sich dies im wesentlichen auf den Staat, insbesondere seinen Sanktionsapparat und seine Legitimität, zurückführen, ergänzt durch die Legitimität des positiven Rechts, das in seinem Kern einen Bestand sozialer Normen gleichläuft und im allgemeinen Bewußtsein mit subjektiven Gerechtigkeitsvorstellungen vermengt wird. Die Häufung staatlicher Sanktionen würde bald an die Grenze totaler Verhaltenskontrolle und Überwachung stoßen. Versagt zudem die vom Staat und vom alten Recht erborgte Legitimität neuen Rechts, so wird entscheidend, daß der Glaube an die Legitimität einer rechtlich kommunizierten Veränderungsabsicht bei den Betroffenen bereits vorhanden ist oder erzeugt werden kann. Wirtschaftlich begründete Machtpositionen oder eine mauernde Bürokratie können die besten Absichten zum Entgleisen bringen. Je stärker die Mächte sind, gegen die positivierte Erwartungen anrennen müssen, um so prekärer ist ihre Durchsetzungschance. Wo diese Erwartungen nicht durch lediglich symbolische Geltung neuen Rechts zufriedengestellt werden, greifen mannigfaltige Strategien der Gesetzesumgehung ein, die das Verhältnis wirtschaftlich oder politisch begründeter Machtpositionen zu politisch-verbal durchgesetzten Forderungen so lange kennzeichnen, wie Forderungen nach sozialer Besserstellung, nach Erhöhung von Lebensqualität, nach Sicherung einer lebenswerten Zukunft mit Profitinteressen und Machtsicherungsinteressen nicht am selben Strange ziehen.

Die Frage nach der Funktion des Rechts bei gesellschaftlichen Veränderungen mündet hier in das allgemeine Problem von oben induzierten gesellschaftlichen Wandels. Solange der Glaube an die Notwendigkeit von Veränderungen sich auf eine kleine

52 Ryffel, Bedingende Faktoren a. a. O., S. 241, Wie sehr Max Webers Einschätzung der legalen Verwaltung diesem Übergangsstadium verhaftet bleibt, wird deutlich bei Martin Albrow, Bürokratie, München 1972, S. $71 \mathrm{ff}$. 
Avantgarde beschränkt, kann der Legalitätsglaube der Bevölkerung und des Beamtenkorps unter bestimmten historischen Bedingungen für eine kurze Übergangszeit den breiten Glauben an die Notwendigkeit einer Veränderung ersetzen. Es ist nicht nur eine Lehre der jüngsten Geschichte, daß hierauf sich zu verlassen tödlich sein kann. Für die Fortentwicklung der Weltgesellschaft ist das entmythologisierte Recht des Obrigkeitsstaats nur noch ein Kommunikationsmittel unter anderen. 
On the instrumental function of law with regard to social change

\section{By Klaus LenK}

Law is often regarded as an instrument for inducing social change by influencing the behavior of its adressees. Such a view is partly due to lack of clearness of the concept of law. Positive law, as enacted by the political power, is not always kept distinct from feelings of justice, social norms, or other phenomena which in many contexts are considered as legal ones.

As an instrument of social change, law is part of the body of instrumental law dealing with social administration in the broadest sense. Unlike the traditional "lawyers' law" - which may also be formally enacted by the State - it is not yet paralleled by actually observed social bhavior. Empirical research on the effectiveness of law indicates that, unless new legal precepts are deemed reasonable by their addressees, their efficacy relies on fears of coercive sanctions, as well as on the legitimacy of the State supporting and enforcing them. To some extent, this legitimacy is strengthened by the new instrumental law being incorporated into the "lawyers' law".

Stressing the role of the law as an instrument of social change was characteristic of the now vanishing normative climate of the "Obrigkeitsstaat" where laws were obeyed simply because they were laws emanating from the State. Such a view is inappropriate for a general assessment of the contribution of law in politically induced processes of social change where the use of legal precepts, while still constituting a privileged means of communication, cannot substitute the broad conviction of the changes to be brought about.

\section{Control and Management of the Economy: Research Perspectives on Public Enterprise}

\section{By Yash GHaI}

The paper suggests some perspectives for research into public enterprise. The main argument is that only a limited understanding of public enterprise can be obtained from a study of the texts of legislation and the case law. It is essential to relate public enterprise to the broader social and political system in which the enterprise functions. Some particular perspectives which are discussed seek to relate the role of public enterprise to the internal class structures of the country in question, as well as to the international context. As to the former, it is argued that public enterprise is used to benefit particular groups in society, and that often their effect may be strength to the private sector activities (and interests) rather than to bring about any kind of socialist transformation. The international context is important because of the heavy dependence of the most developing countries on the Western group of countries, and because of the argument that a primary cause of the backwardness of the developing countries is their exploitation by the industrialised countries. Public enterprise provides a useful vehicle through which to examine the links and the relationships between the international capitalist system 